\title{
FDG-PET in paraneoplastic limbic encephalitis
}

\author{
F. Troester • G. Weske • E. Schlaudraff • B. Passlick • \\ K. Kraemer
}

Received: 9 September 2008 / Accepted: 23 October 2008 /Published online: 24 December 2008

(C) Springer-Verlag 2008

Limbic encephalitis (LE) is characterized by a subacute onset of impaired cognitive function, temporal lobe epilepsy, and psychiatric symptoms. Common causes of LE are autoimmune disorders, among which paraneoplastic findings are frequent $[1,2]$. The neurological symptoms usually precede the detection of the primary cancer. We report on the imaging findings of paraneoplastic LE in a 58year-old patient. Clinical findings included subacute loss of memory function, seizures, and weight loss of $15 \mathrm{~kg}$. In a search for an underlying malignancy, chest radiography was inconclusive. A CT scan of the chest (a) and a FDG-PET scan (b) showed a hypermetabolic tumour (arrows) in the right hilar region. The FDG-PET scan also detected a mediastinal lymph node metastasis (arrowhead). The FDGPET scan of the brain (c transverse and coronal slices) showed bilaterally increased FDG uptake in the temporomesial cortex, a typical sign of LE [3]. These findings were underlined by 3-D stereotactic surface projection (NEUROSTAT/3D-SSP; University of Washington, Seattle. WA) of the brain scan (d top) [4]. Compared to a normal database (d bottom), FDG uptake in the temporomesial cortex was increased by up to seven standard deviations. The final histological diagnosis was small-cell lung cancer (pT1, pN2 (3/28), cM0).

\section{F. Troester $(\bowtie) \cdot$ K. Kraemer}

Department of Nuclear Medicine, University Hospital Freiburg,

Hugstetter Str. 55,

79106 Freiburg, Germany

e-mail: fridolin.troester@pluto.uni-freiburg.de

\section{G. Weske}

Department of Neurology and Neurophysiology,

University Hospital Freiburg,

Freiburg, Germany

\section{E. Schlaudraff}

Department of Radiology, University Hospital Freiburg,

Freiburg, Germany

\section{B. Passlick}

Department of Thoracic Surgery, University Hospital Freiburg, Freiburg, Germany
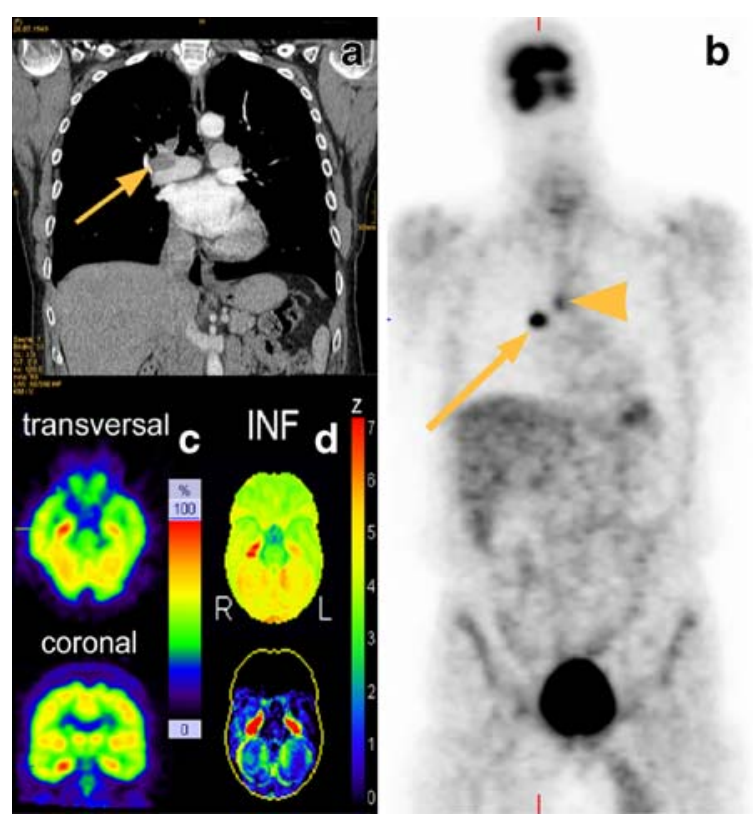

If paraneoplastic LE is suspected, FDG-PET may contribute to the early detection of an underlying malignancy and simultaneously confirm the clinical diagnosis of LE by showing temporomesial hypermetabolism [3].

\section{References}

1. Tüzün E, Dalmau J. Limbic encephalitis and variants: classification, diagnosis and treatment. The Neurologist 2007;13:261-71.

2. Gultekin SH, Rosenfeld MR, Voltz R, Eichen J, Posner JB, Dalmau J. Paraneoplastic limbic encephalitis: neurological symptoms, immunological findings and tumour association in 50 patients. Brain 2000;123:1481-94.

3. Ances BM, Vitaliani R, Taylor RA, Liebeskind DS, Voloschin A, Houghton DJ, et al. Treatment-responsive limbic encephalitis identified by neuropil antibodies: MRI and PET correlates. Brain 2005;128:1764-77.

4. Minoshima S, Ficaro EP, Frey KA, Koeppe RA, Kuhl DE. Data extraction from brain PET images using three-dimensional stereotactic surface projections. In: Carson RE, Herscovitch P, DaubeWitherspoon ME, editors. Quantitative functional brain imaging with positron emission tomography. San Diego, CA: Academic Press; 1998. p. 133-7. 